\title{
USING SIMULATION FOR STUDYING THE EFFECT OF ORIENTATION ON THE THERMAL PERFORMANCE OF SPACES IN RESIDENTIAL BUILDINGS (NEW ASSIUT CITY AS A CASE STUDY)
}

\section{Ahmed Abd El-Monteleb Mohammed Aly}

Tutor, Department of Architecture, Faculty of Engineering, Assiut

University, Egypt. ahmed.monteleb@hotmail.com

\section{Essam El-Deen K. Mahroos}

Professor of Urban Design,Department of Architecture, Faculty of

Engineering, Assiut University, Egypt dr essam mahrous@yahoo.com

\section{Ezzat A. Morghany}

Associate Professor of Architectural Design, Dept. of Architecture, Faculty of

Engineering, Assiut University, Egypt ezzatmorghany@yahoo.com

\section{Essam Salah Saeed}

Lecturer of Architectural Design, Dept. of Architecture, Faculty of Engineering,

Assiut University, Egypt. $\quad$ essam_sss@yahoo.com

(Received June27, 2011 Accepted July 26, 2011)

Research, in the field of studying the effect of orientation on the ambient temperature of residential buildings, is still in its early phases despite the leaps made by studies and experiments. Most climatic studies focused on studying residential buildings as to: orientation, building materials use, thermal behavior, etc. This was done by theoretical studies and few on-site measurements. Studying orientation of residential buildings by simulation is rare in the Arab world.

The present generation of climatic design tools should rely more on digital presentation by computers, in order to aid designers make sound design decisions based on visible results. Thus, the computer carries out all calculations relieving designers from their heavy burden.

Due to the scarce use of simulation software in the climatic assessment of residential buildings; the study aims at studying the effect of orientation on the thermal performance of spaces in residential buildings at New Assiut City, Egypt.

To achieve this aim, the research is done by the analytical and applied methods, using the simulation software Thermal Analysis Software, from the climatic analysis of New Assiut City - as well as identifying the prevailing residential patterns, and detailed study of the selected residential building, identifying the software used, and analyzing the simulation results for ambient room temperatures of the cold and hot periods - for different orientations of the selected model. The research ends with a number of results and recommendations. Such results may be applied in hot desert areas. 
استخدام المحاكاة لاراسة تأثير التوجيه علي الأداء الحراري لفراغات المباني السكنية (مدينة أسيوط الجيديدة كمثال)

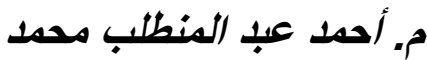

معبد بقسم الهندسة المعمارية

قسم العمارة- كلية الهندسة - جامعة أسيوط - مصند

\section{لـ د. عصام صلاح سعبل}

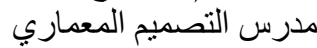

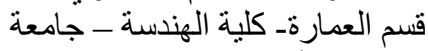

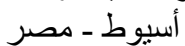

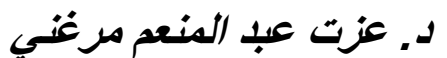

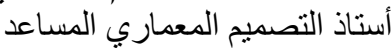

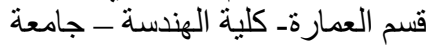
أسيوط - مصر المندر

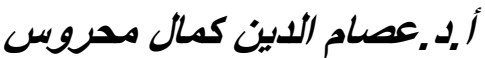

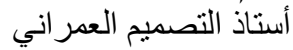

قسم العمارة- كلية الهندسة - جامعة العية

أسيوط - مصر - مندر

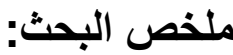

تعد دراسـات تأثبر التوجيه على حرارة البيئة الداخلية للمباني السكنية غبر كافبية رغم الخطوات التهي قطعتها

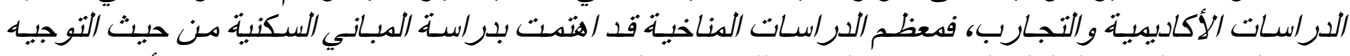

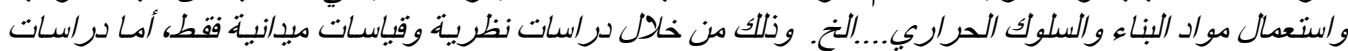

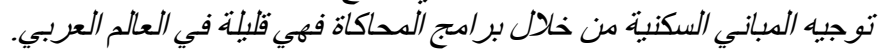

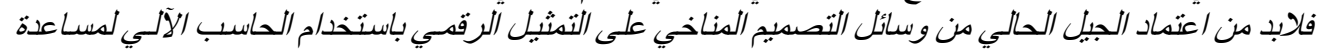

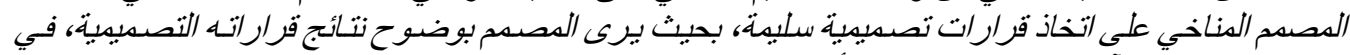

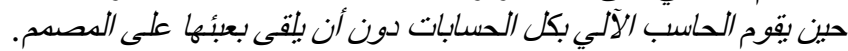

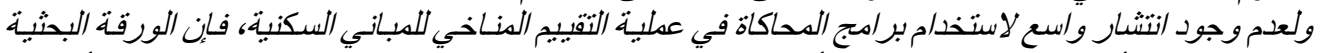

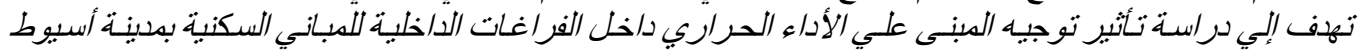

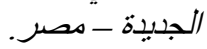

لتحقيق هدف الدراستة بعتهد البحث علي المنهج التحلبلي والتطبيقي باستخدام برنامج محاكاة وهو من Analysis $\underline{\text { Software }}$

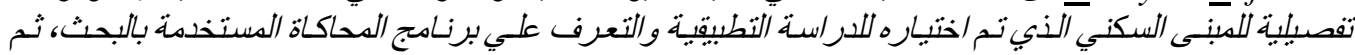

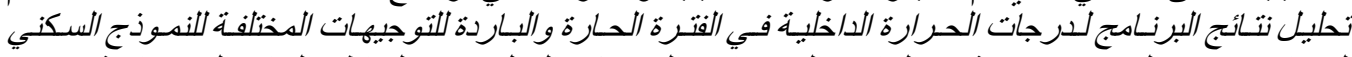

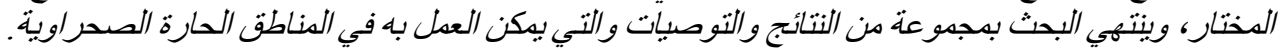

ثقدمبـم:

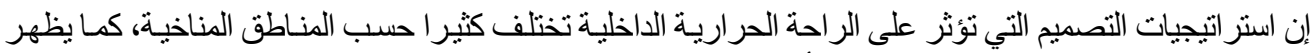

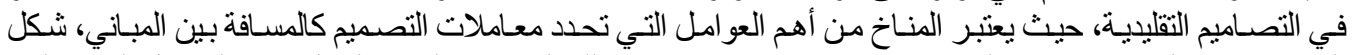

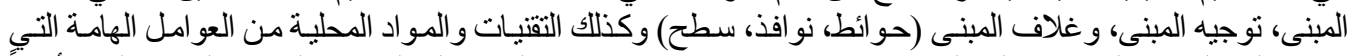

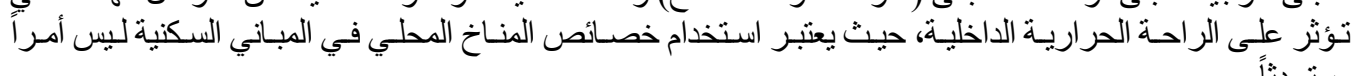

لذا نجد أنه يمكن تجنب الكثير من المشـاكل بالعمل الدقيق أثناء تطور مر احل التصميم لتقليل آثار العو امل المناخية

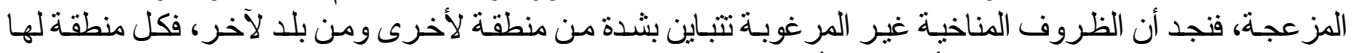

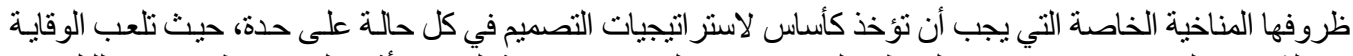
من الثمس والحرارة دورا هأما في المناطق التي يرتفع بها الفارق بين درجة الحرارة أنثاء النهار ونظيرتهات التها في الليل.

\section{إثكالية الدراسة:}

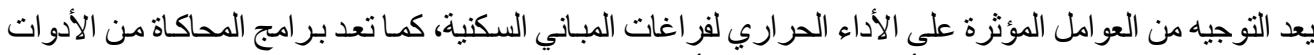

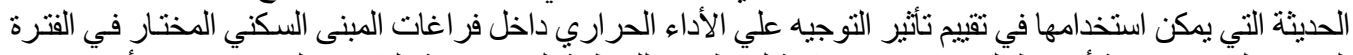

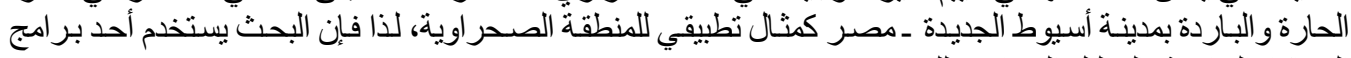




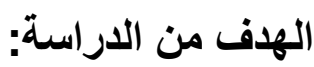

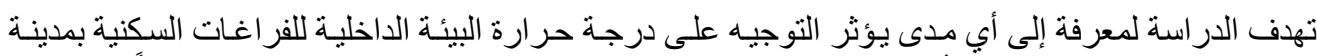

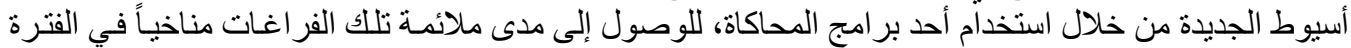
الحارة و الباردة من السنة.

\section{منهجية الدراسة:}

لتحقيق هدف الدر اسة يعتمد البحث على المنهج التحليلي و التطبيقي من خلال:

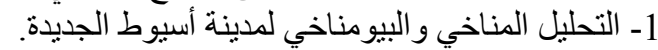

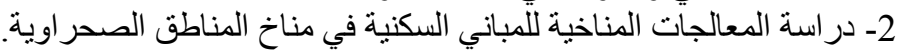

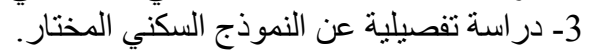

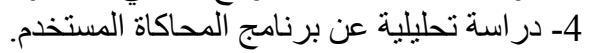

5- تحليل نتائج البرنامج لدرجات الحر ارة في الفترة الحارة و الباردة للمبنى السكني المختار.

\section{1- التحليل المناخي لمدينة أسيوط الجديدة:}

تقع مدينة أسيوط الجديدة شرق نهر النيل على طريق القاهرة سوهاج الصحر اوي عند التقائه مع طريق الغردقة

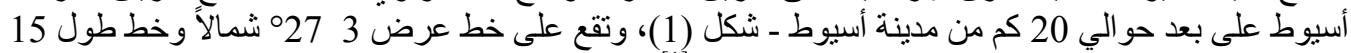

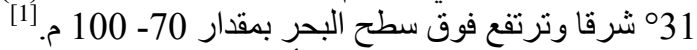

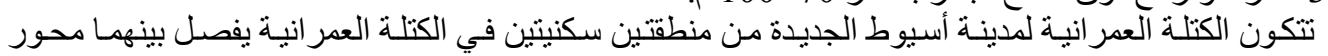

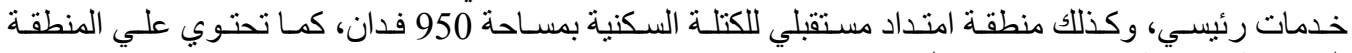
الصناعية بمساحة 180 فدان - شكل (2).

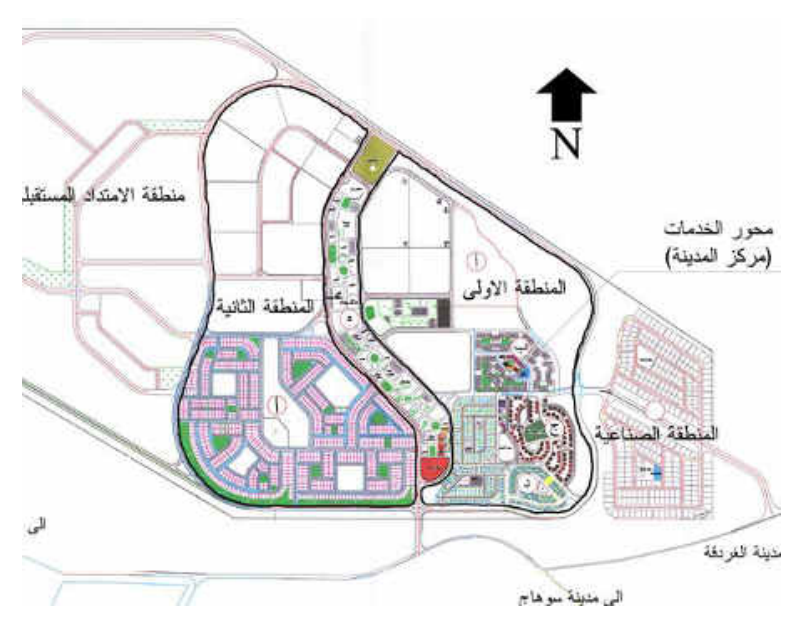

شكل (2): الموقع العام لمدينة أسيوط الجديدة. [2]

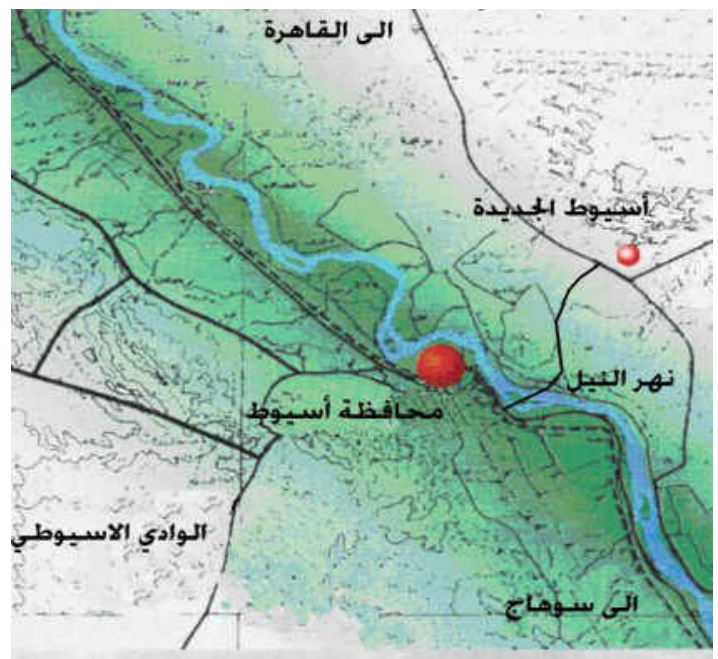

شكل (1): يوضح موقع مدينة أسيوط الجديدة بالنسبة

للمحافظة. [1]

$\left.{ }^{1}{ }^{1}\right]$ Tarek Galal Habib, Trains of Urban Development in Egypt, Update Evaluation for the Experience of New Urban Communities, Ph. D., Faculty of engineering, University of Assiut, 2000, p 126.

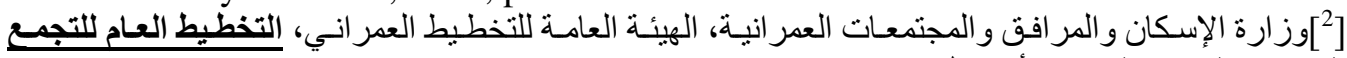

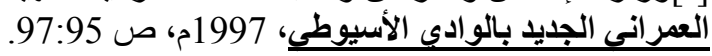


يتم التحليل المناخي من البيانات التي أمكن الحصول عليها من هيئة الأرصـاد الجويـة،1] وفي مـا يلي عرض تحليلي لتلك البيانات:

\section{1-1-1 سطوع الثمس والإثُعاع الثمسي:}

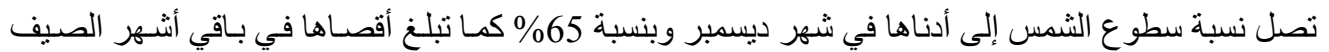

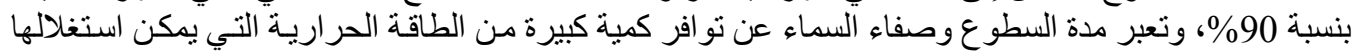
في تدفئة ليالي الثتاء الباردة.

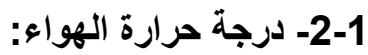

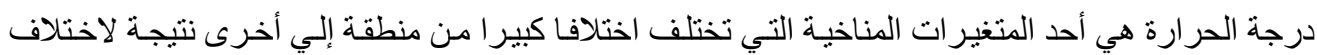

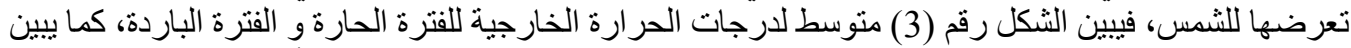

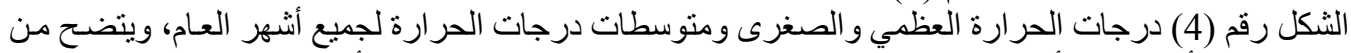

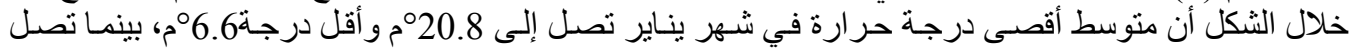

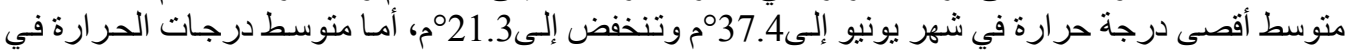

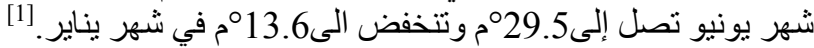

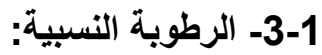

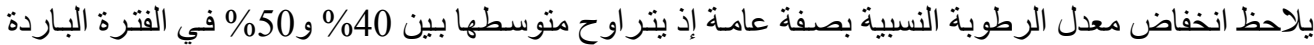

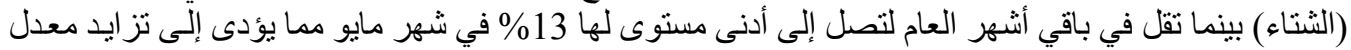

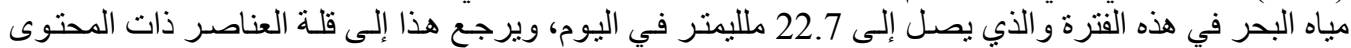

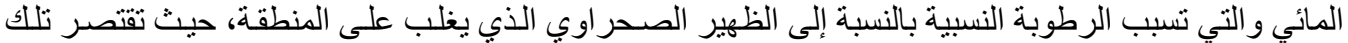

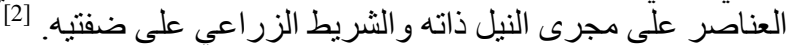

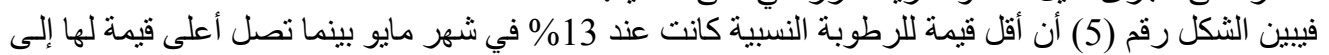

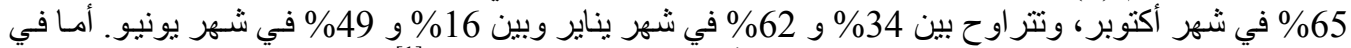

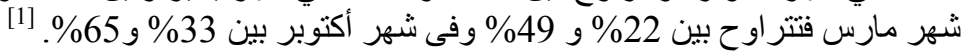

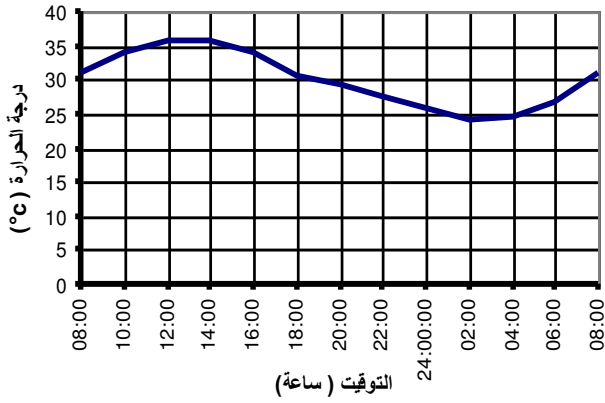

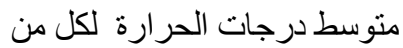

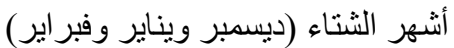

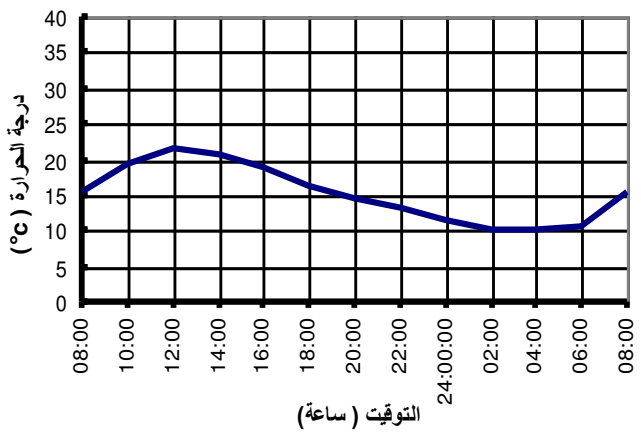

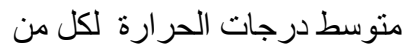
أشهر الصيف (يونيو ويوليو وان أغرأ أغسطس)

شكل (3): يوضح متوسط درجات الحرارة الخارجية لكل من أثهر الصيف وأثهر الشتاء لمدينة أسيوط الجديدة.

[ [1] الهيئة العامة للارصداد الجوية، الأطلس المناخى لمصر، وزارة النقل و المو اصلات، جمهوريـة مصر العربية، 1996

[2] [وزارة الإسكان و المر افق و المجتمعـات العمر انيـة، العيئة العامـة للتخطبط العمر انـي، التخطيط العـام للتجمـع

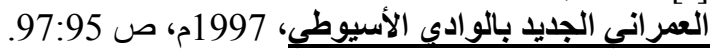




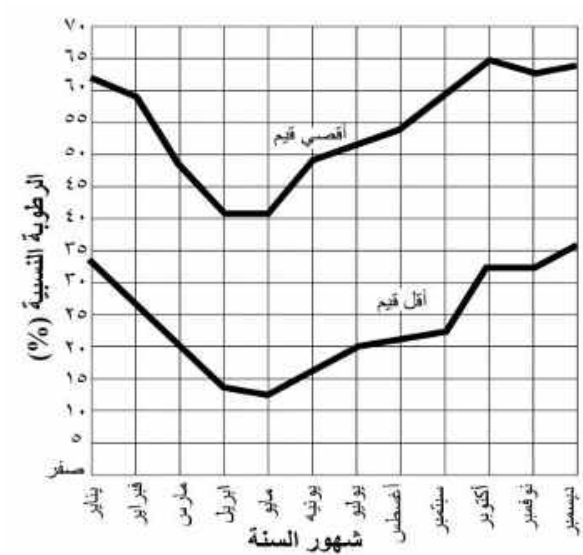

شكل (5): يوضح متوسط أقصى و أقل درجات الرطوبة الربة

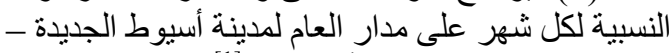
الو ادي الأسيوطي.

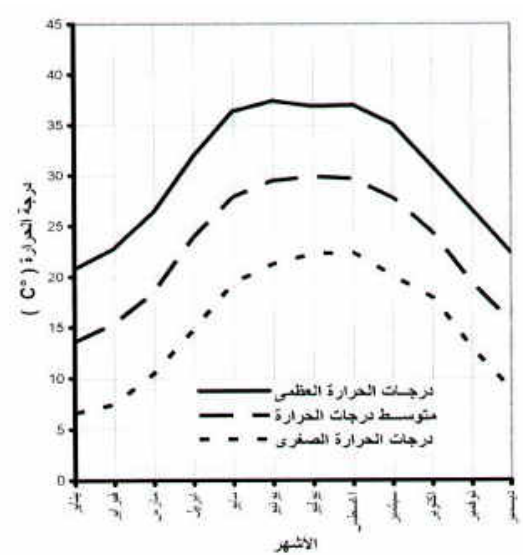

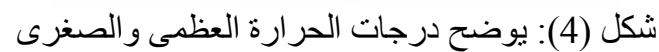

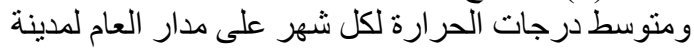
أسيوط الجديدة. [1]

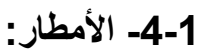

يصل مجموع كمية المطر في أثشهر السنة إلى أقصـاه في شـهر فبراير، حيث يبلغ 0.4 ملليمتر ويمتد موسم

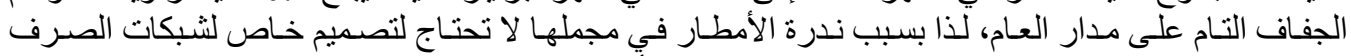

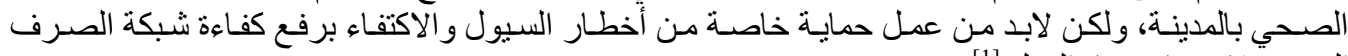

الصحي لاستيعاب مياه السبل.

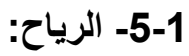

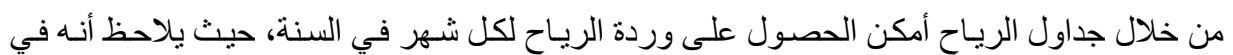

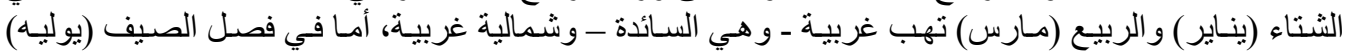

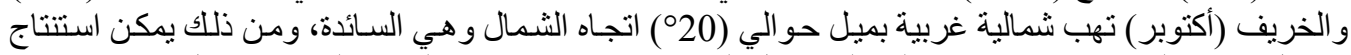

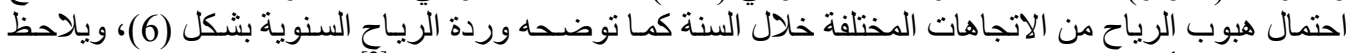

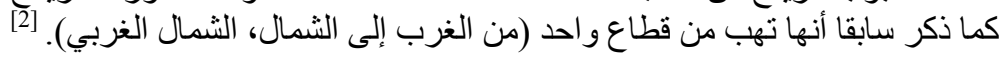

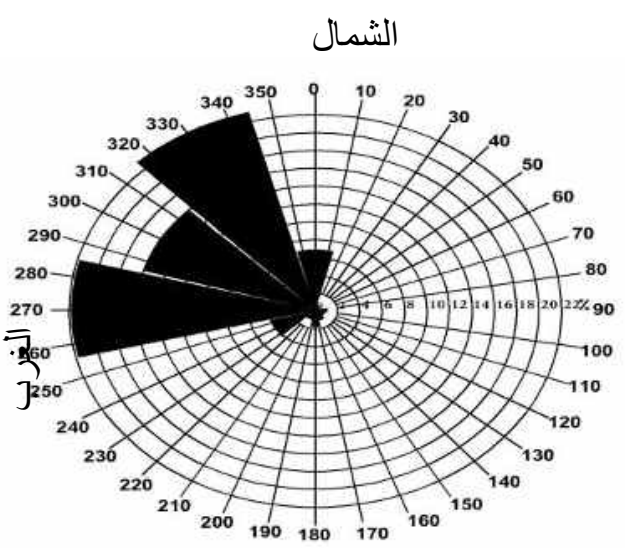

$$
\text { شكل (6): يوضح وردة الرياح السنوية أسيوط الجديدة. }
$$

[ [1] الهيئة العامة للارصـاد الجوية، الأطلس المناخى لمصر، وزارة النقل و المو اصلات، جمهوريـة مصر العربية، 


\section{2- دراسة المعالجات المناخية للمباني السكنية في مناخ المنناطق الصحراوية:}

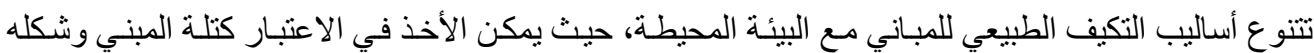

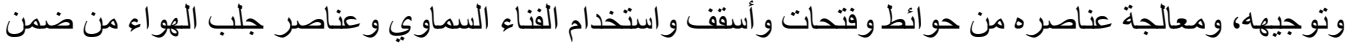

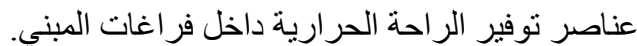

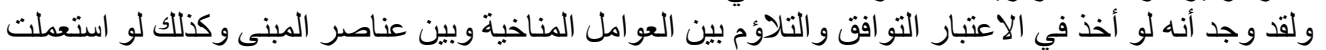

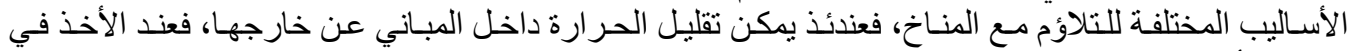

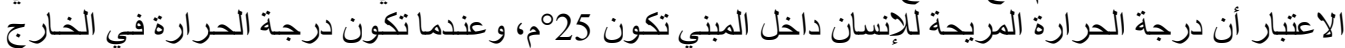

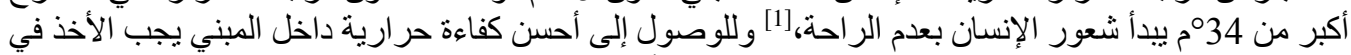

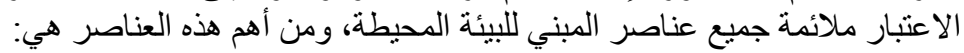

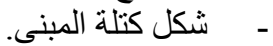

-

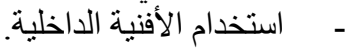

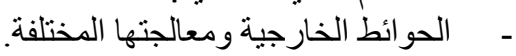

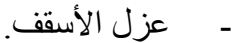

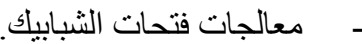

- - كاسر ات الثمس الأفقية و الر أسية و المركبة و غير ها.

ويتناول البحث هنا شرح لعنصر واحد فقط وهو التوجيه وتأثيره علي تحسين الأداء الحراري داخل الفراغات

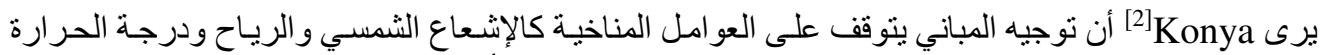

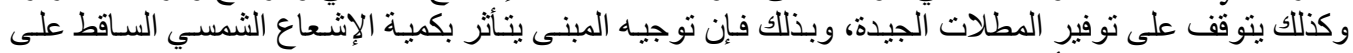

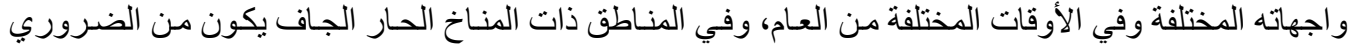

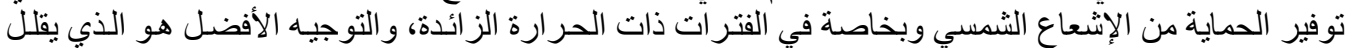

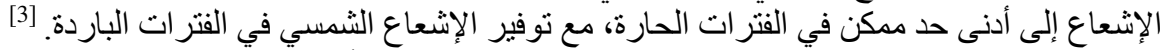

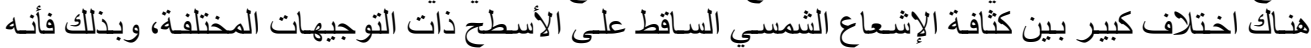

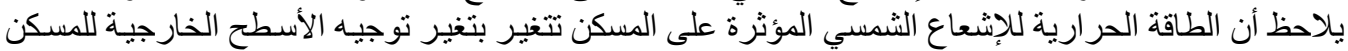

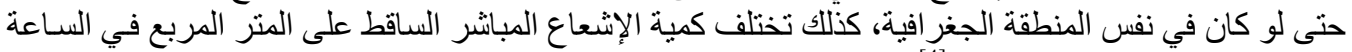

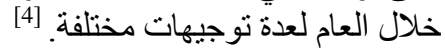

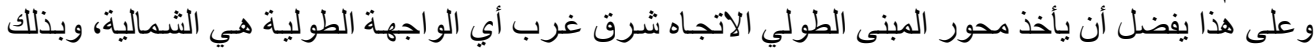

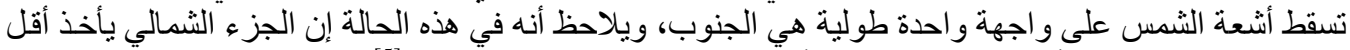

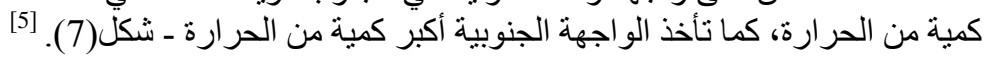

[1] نيفين يوسف عزمي يوسف، الإشعاع الثمسي والنسيج العمرانى (مدخل لتشكيل طرق متو افقة مـع الإشعاع

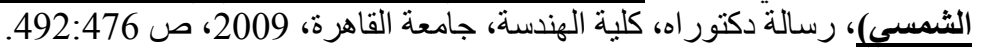

${ }^{[2]}$ Konya,A.: Design Primer for Hot Climates, The Architectural Press Ltd, London, 1980, P,37.

${ }^{[3]}$ Salwa Abdel Moneim El-Ginidy, The Effect of Building Envelope Design On Energy Conservation Op Cit., p 224:230.

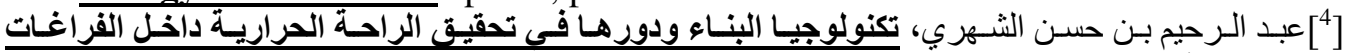

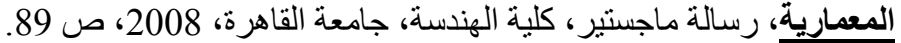

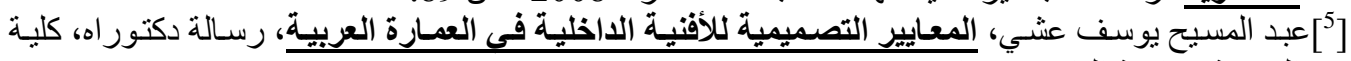
الهندسة، جامعة القاهرة، فبر اير المعبد 1999، ص ص 127. 


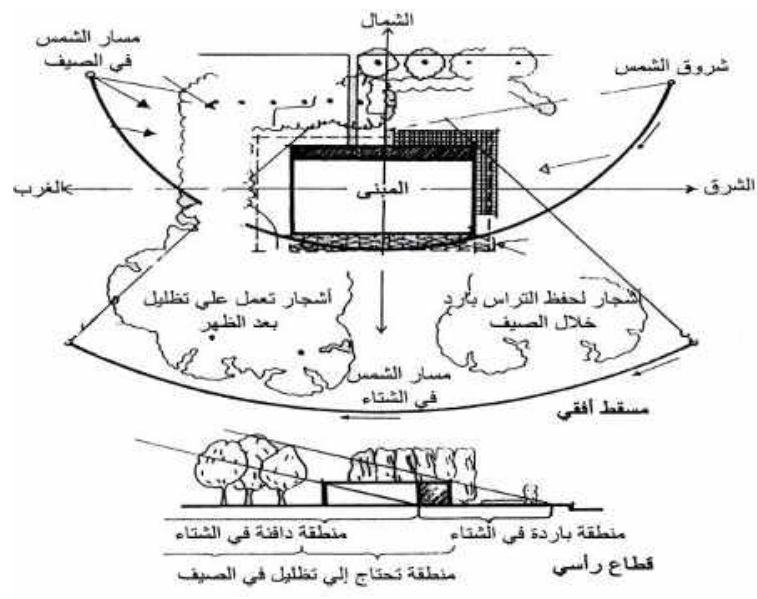

شكل (7): يوضح توجيه المبنى وكيفية حمايته من الثمس في الصيف باستعمال وسئل

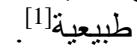

\section{3- دراسة تفصيلية عن النموذج السكني المختار:}

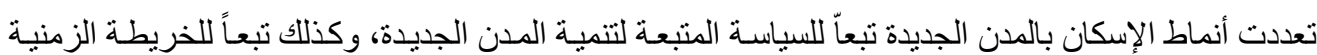

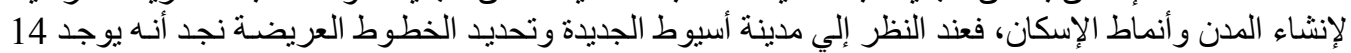

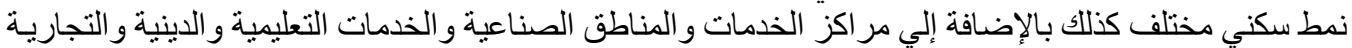
و امتداد لجامعة أسيوط وهم كالآتي:

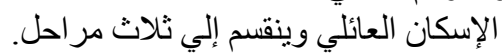

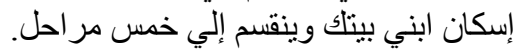

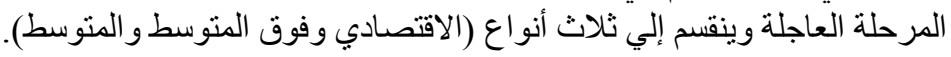
إسكان الشباب و إسكان المستقبل. الإسكان المطور. المبكان الإسكان القومي وينقسم إلي مرحلتين. حي الز هور. الإن الزعي

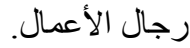
إسكان استثماري ومنطقة الفيلات.

ولتحديد النمط السكني لدر استه وتحليل نماذجه السكنية، كان لابد من حساب مساحة كل نمط سكني وكذلك نسبته الإنه

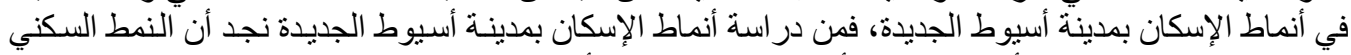

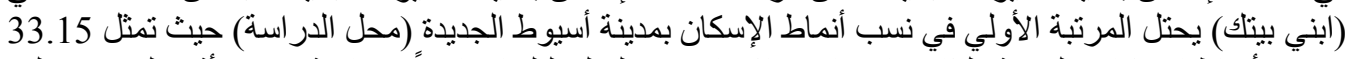

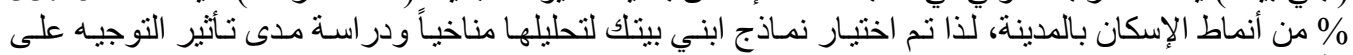

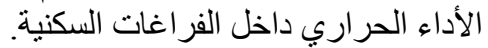

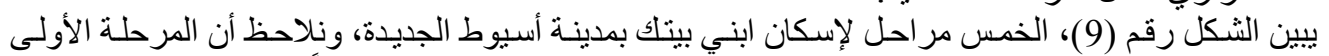

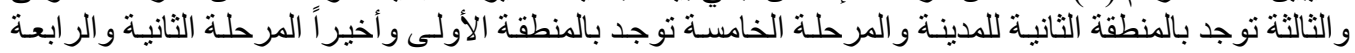

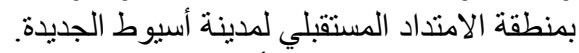

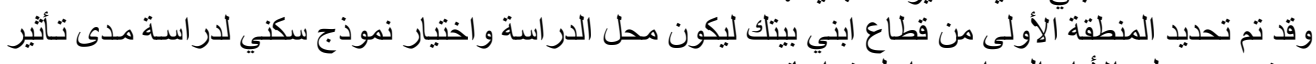

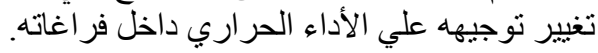

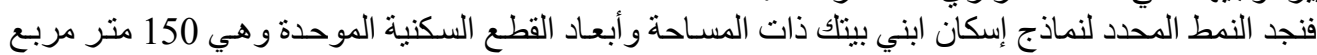

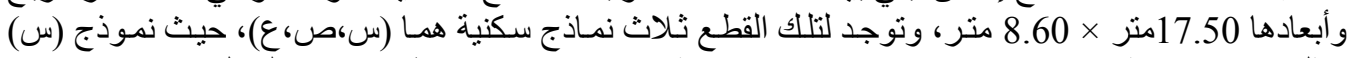

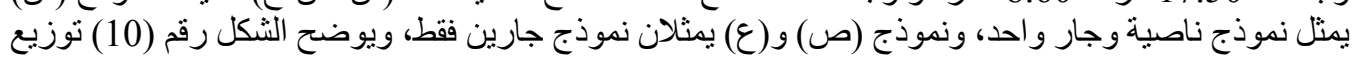

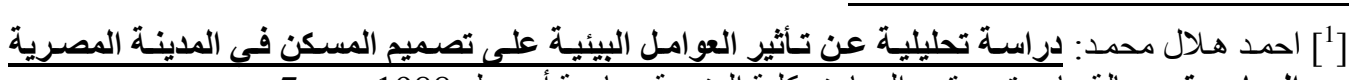

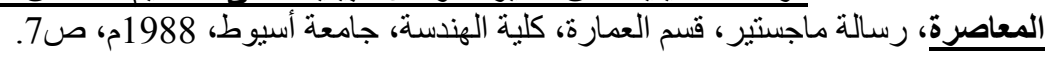


الثلاث نماذج (س،ص،وع) الخاصة بالمرحلة الأولى بقطاع ابني بيتك بمدينة أسبوط الجديدة، ومنها أمكن تحليل

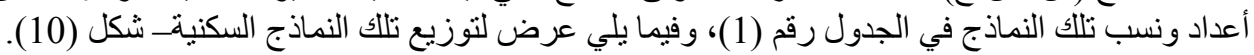

جدول (1): يوضح أعداد ونسب الثثلاث نماذج بابني بيتك

\begin{tabular}{|c|c|c|}
\hline النسبة & العدد & النموذج \\
\hline$\% 40.95$ & 206 قطعة أرض & نموذج (س) \\
\hline$\% 2.60$ & 13 قطعة أرض & نموذج (ص) \\
\hline$\% 56.45$ & 284 قطعة أرض & نموذج (ع) \\
\hline
\end{tabular}

لذا فقد تم اختبار النموذج الثالث وهو (ع) لتقييم الأداء المناخي له وتحسين الأداء الحر اري للفر اغات بـه. 


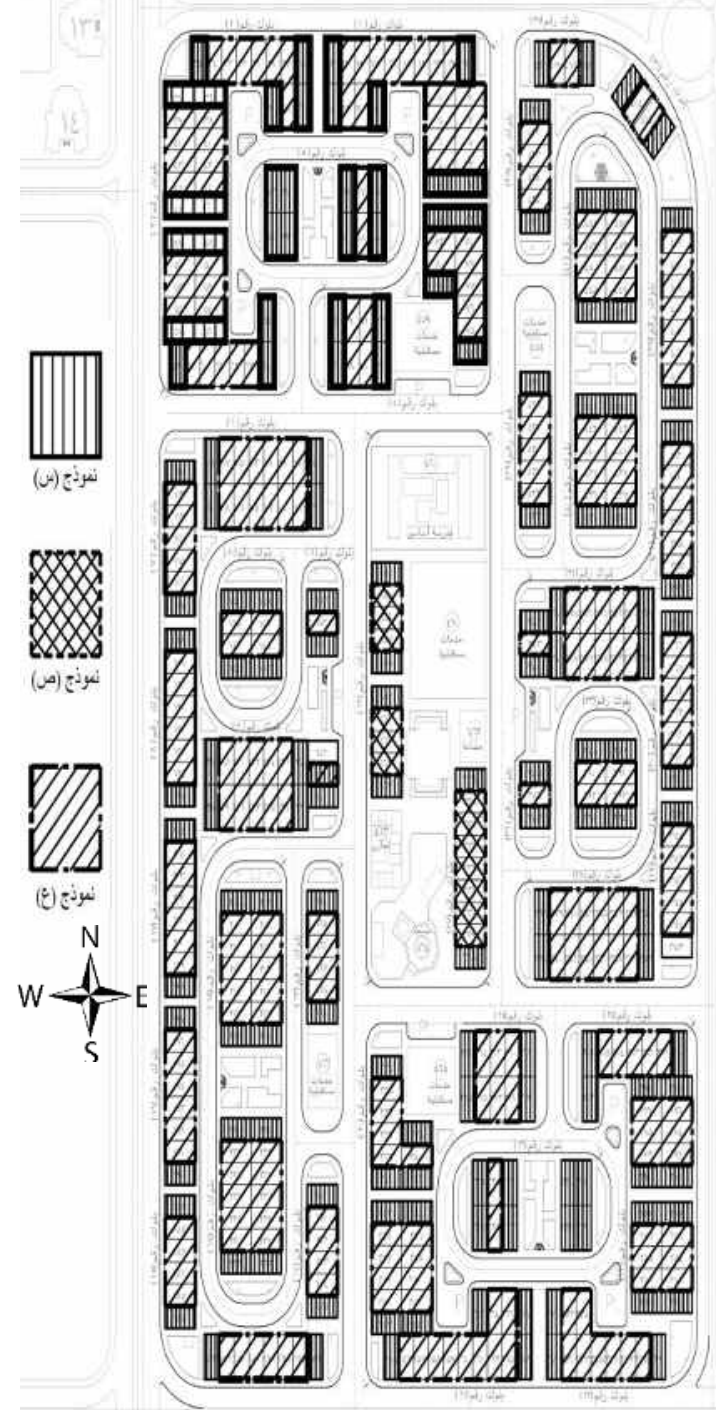

شكل (9): يوضح مر احل تنفيذ ابني بيتك بمدينة أسيوط شكل (10): يوضح توزيعة نماذج المرحلة الأولى بإسكان ابني [ [مز ارة الإسكان و المر افق و المجتمعات العمر انية، هيئة المجتمعات العمر انية الجديدة، مدينة أسيوط الجديدة. 
ويوضح شكل (11) المساقط الأفقية والو اجهات للنموذج الثالث (ع) المختار - نموذج الجارين.

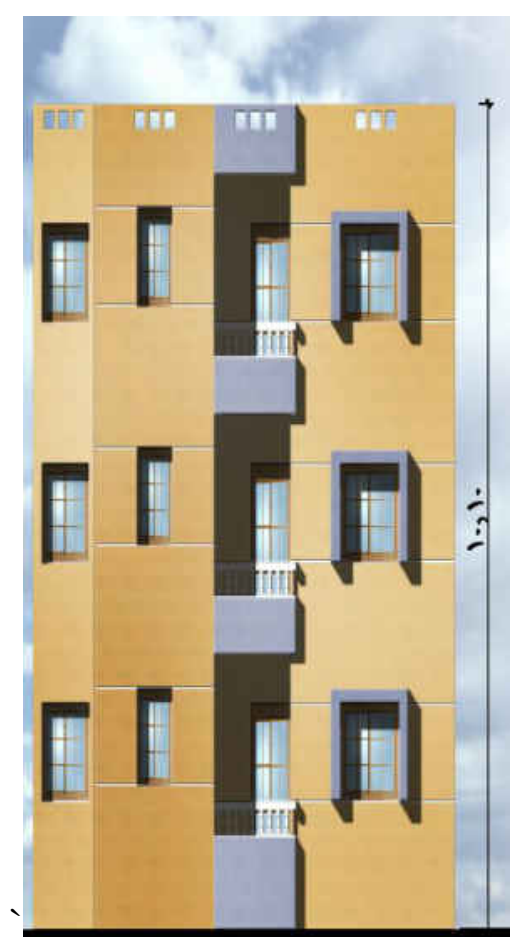

الو اجهة الخلفية لنموذج (ع) - جارين

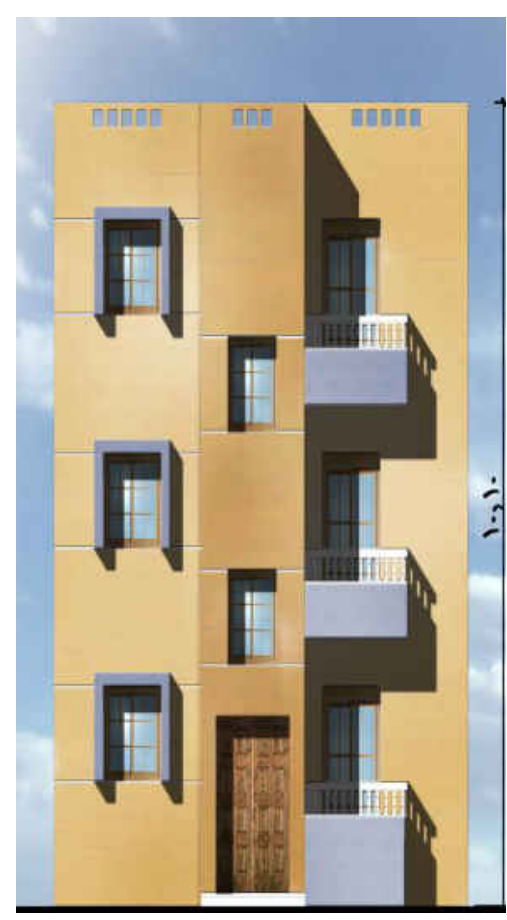

الو اجهة الأمامية لنموذج (ع) - جارين

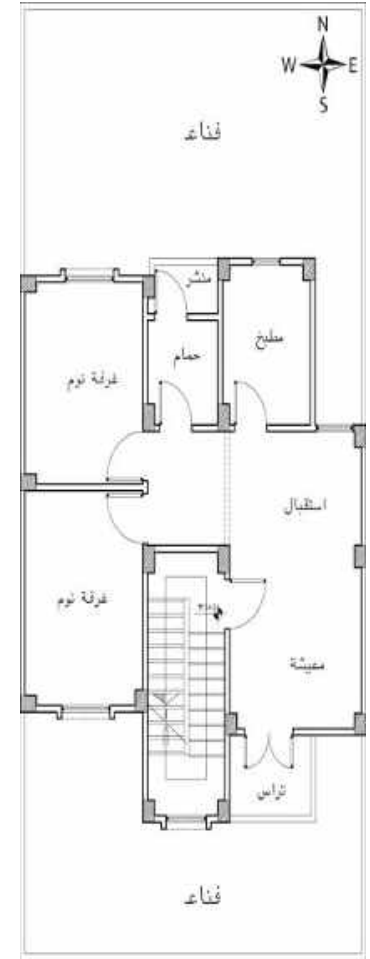

المسقط الأفقي للاور المتكرر [1]

شكل (11): يوضح المساقط الأفقية والواجهات لنموذج (ع) بقطاع ابني بينك بأسيوط الجديدة

4- نبذة عن برامج المحاكاة المستخدمة في دراسة الأداء الحراري داخل المباني:

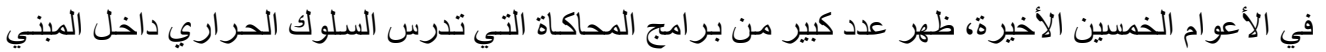

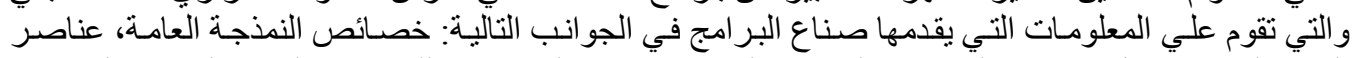

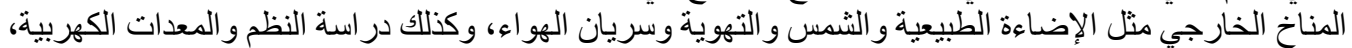

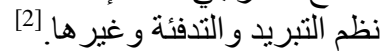

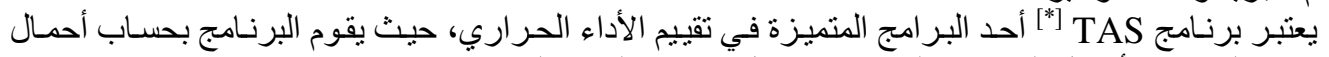

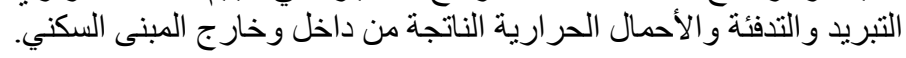

1-4- نبذة عن برنامج المحاكاة المستخلم (TAS):

[1] [وز ارة الإسكان و المر افق و المجتمعات العمر انية، هيئة المجتمعات العمر انية الجديدة، مدينة أسيوط الجديدة. [2]Drury B. Crawley, Jon W. Hand, Michae“ 1 Kummert, Brent T. Griffith, Contrasting the capabilities of building energy performance simulation programs, Building and Environment, V(43), (2009), pp. 661:677. 


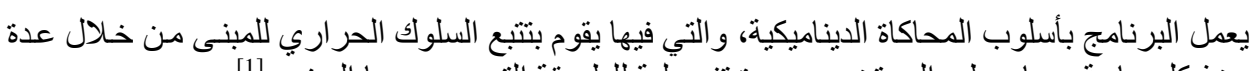

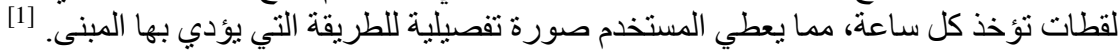

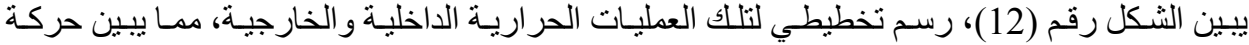

الحر ارة في مختلف الأشكال من حيث توصيلها من و إلى المبنى عبر آليات انتقال الحرارة الثيل المختلفة.

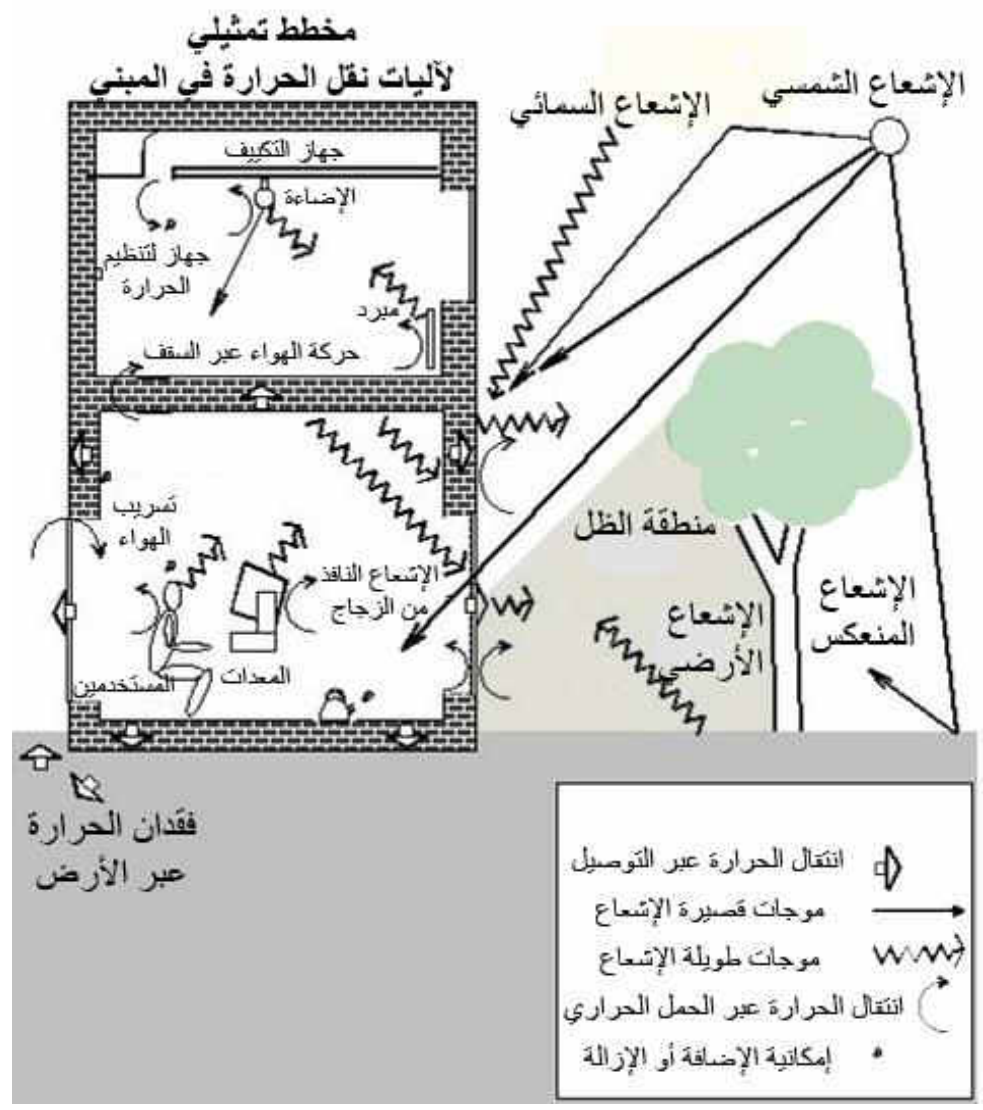

شكل (12): تيوضح تأثنير

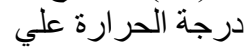
الغلاف الخارجي للمبني

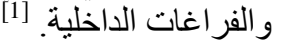

يعتبر برنامج

أيضا Tas3D) ويتكون البرنامج من ثلاث مكونات رئيسية و أساسية وهم:

TAS 3D Modeler, TAS Building Simulator, TAS Results Viewer

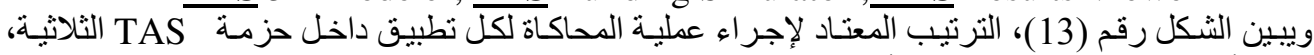

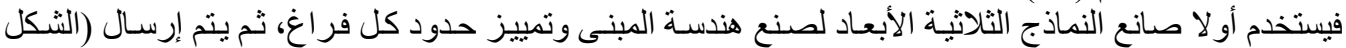

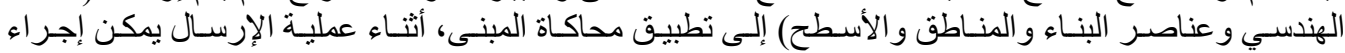
الحسابات المختلفة.

$\left.{ }^{1}\right]$ James Y. P. Lee, BASc, LEED AP, SUSTAINABLE SOLUTION TO BUILDING MECHANICAL SYSTEM - SIMULATION OF THERMO-ACTIVE SLAB WITH THERMAL MASS USING, TAS Earth Tech Canada Inc., Global Facility and Infrastructure, Mechanical Engineering Vancouver, British Columbia, Germany, 2010, pp. 10.

$\left[{ }^{2}\right]$ http://www.edsl.net/main/Support/Documentation.aspx 


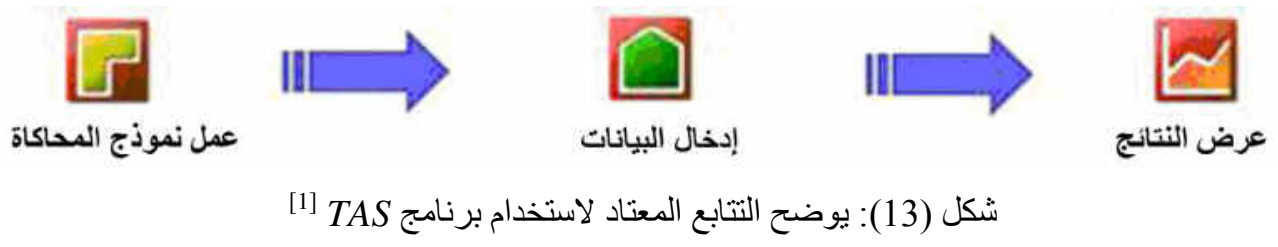

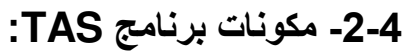

و عند النظر إلي نافذة البرنامج فإنها تضم أو امر إعداد ورسم المبنى وعمل فتحات الأبو اب و الثبابيك ووسائل

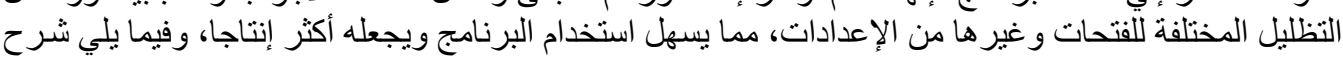
لتلك المكونات:

\section{1-2-4 بناء النموذج السكني (محل الاراسة):}

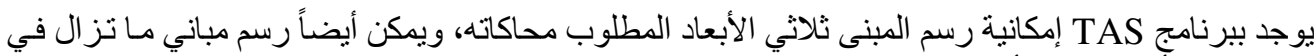

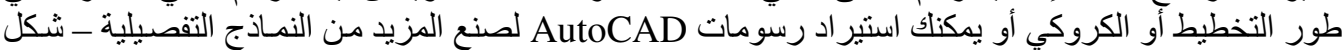

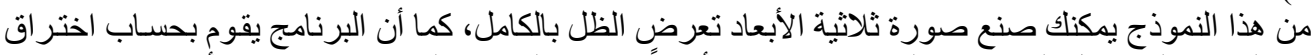

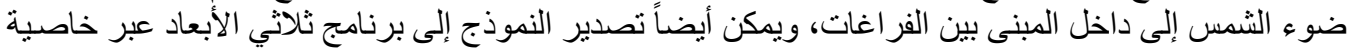

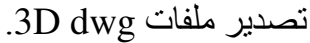

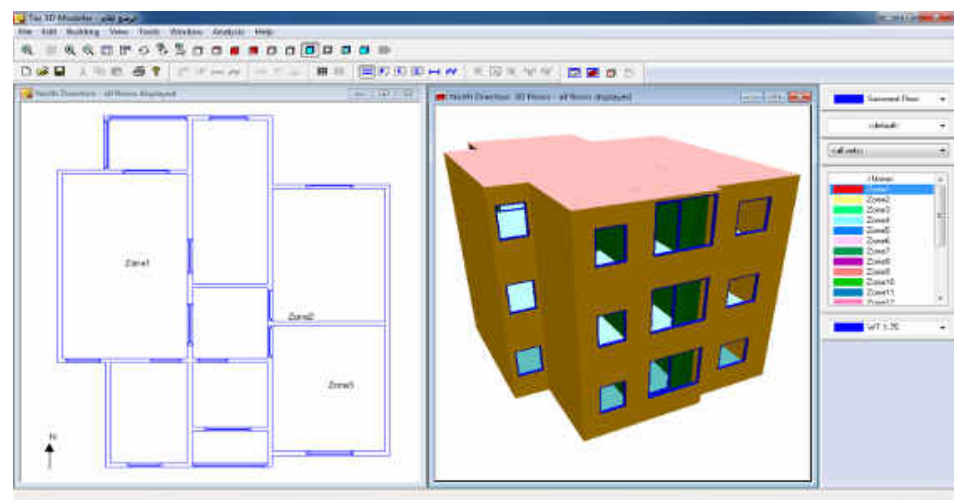

شكل (14): يوضح نافذة

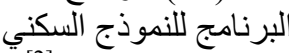
المطلوب محاكاته [2 المبني

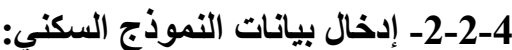

يتم إدخال جميع بيانات النموذج السكني وهي كالنالي: (البيانات المناخية للمنطقة وإدخال سـاعات إثـالئال المبنى

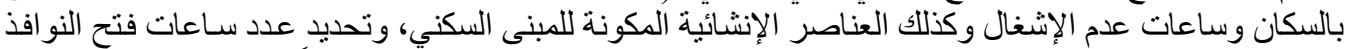

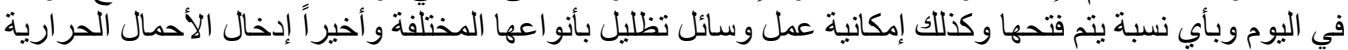

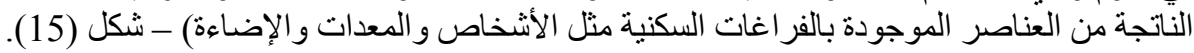

$\left.{ }^{1}\right]$ TAS Building Designer software (EDSL Tas Version 9.2.0) 

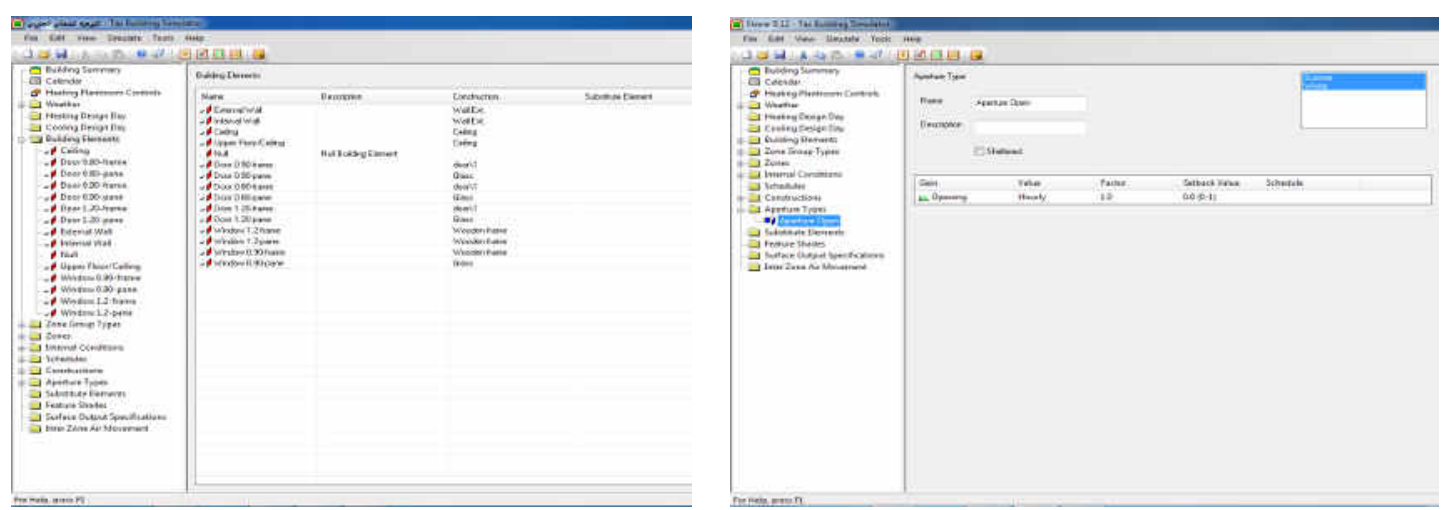

شكل (15): يوضح نافذة إدخال بيانات النموذج السكني المطلوب محاكاته [1]

3-2-4 عرض نتائج برنامج المحاكاة:

يمكن عرض أي مجمو عة معاملات من أي عدد من المناطق و الأسطح ومقارنتها في صورة جداول ومنحنيات.

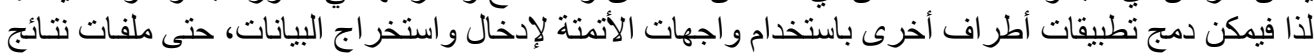

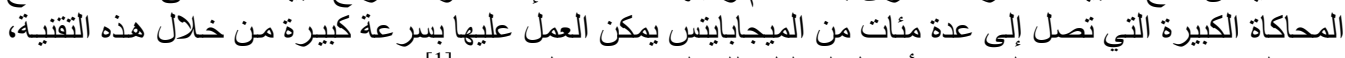

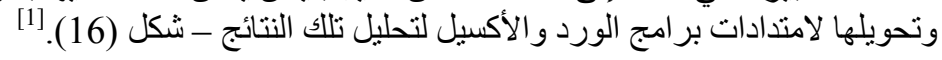

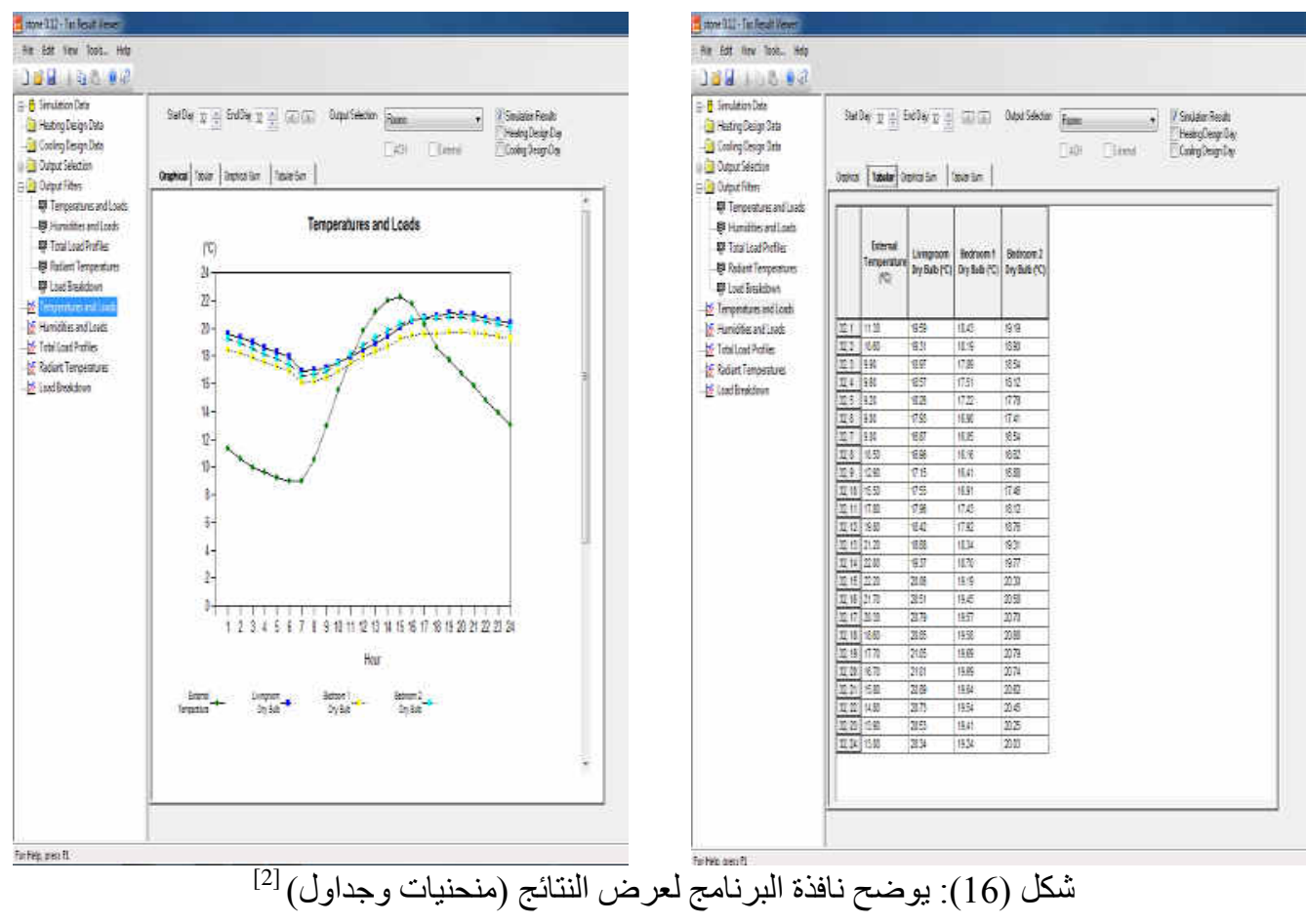

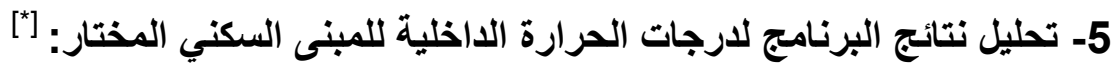

[ $\left.{ }^{1}\right]$ http://www.edsl.net/main/Support/Documentation.aspx

[2]TAS Building Designer software (EDSL Tas Version 9.2.0)

[*]تم معايرة دقة نتائج برنامج الححاكاة بدر اسة سابقة وكان معامل تصحيح النتائج من 2,00 - 2,50 \%. 
تتم عملية المحاكاة علي نموذج (ع) السكني المختار سابقاً، ومنها يمكن الحصول على متوسط درجات الحرارة

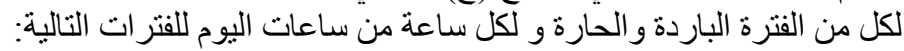

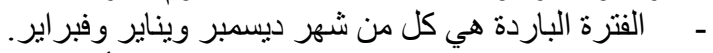

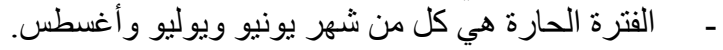

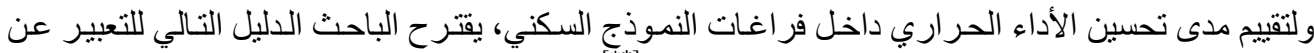

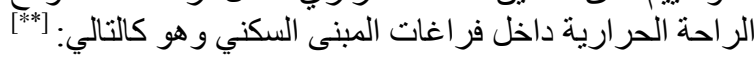

T.C.I $=\left(\mathrm{T}_{\text {Mean } \mathrm{W}} / \mathrm{T}_{\text {Mean S }}\right) \times 100$

T.C.I $=$ Thermal Comfort Index

حيث:

$\mathrm{T}_{\text {Mean } \mathrm{W}}=$ Mean Temperature for Winter

$\mathrm{T}_{\text {Mean } \mathrm{S}}=$ Mean Temperature for Summer

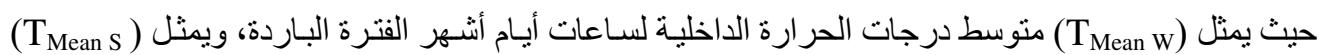

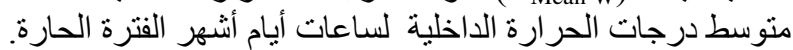

لتحسين الأداء الحراري دارة داخل الفر اغات لابد من الحصول علي أكبر قيمة لهذا الدليل، ويتم دراسة تأثير التوجيه

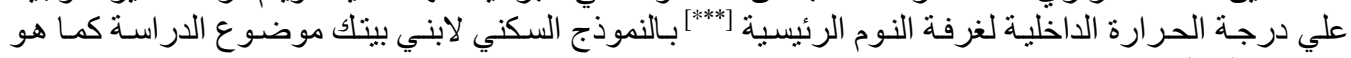
موضح بالثكل (17). 
شارع بعرض • 1 مثر

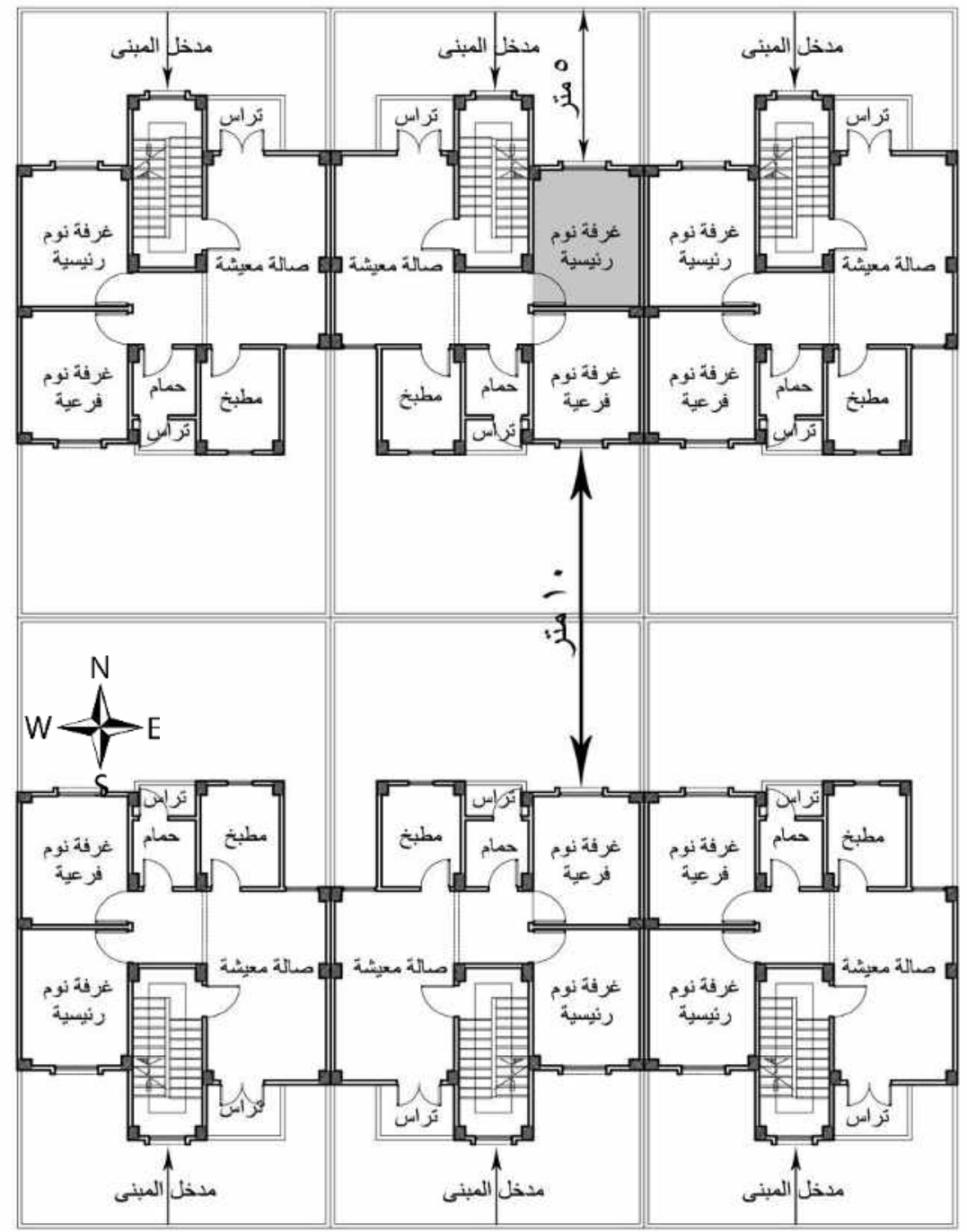

شكل (17): يوضح النموذج السكني لابني بيتك موضوع الدراسة

1-5 تأثثر تغيير توجيه النموذج السكني في كل من الفترة الباردة والحارة:

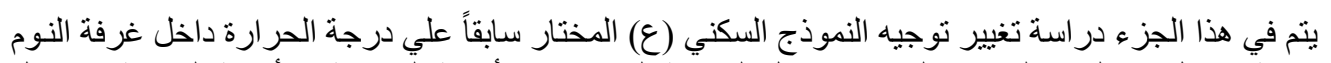

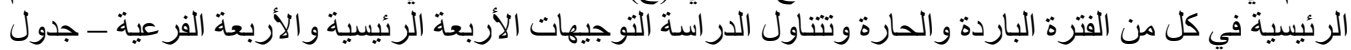

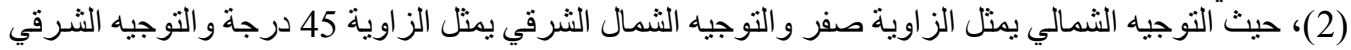

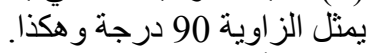

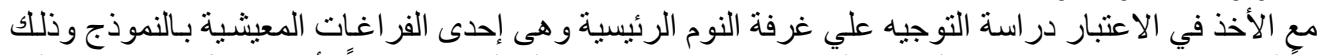

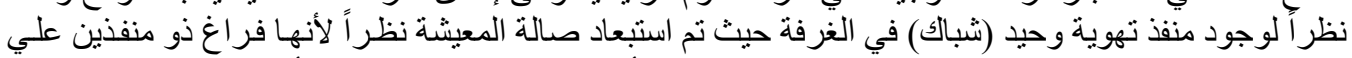

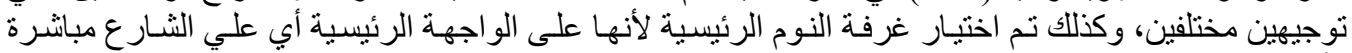

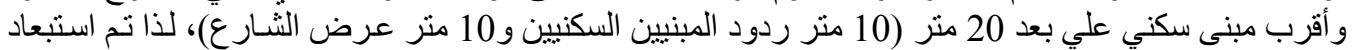

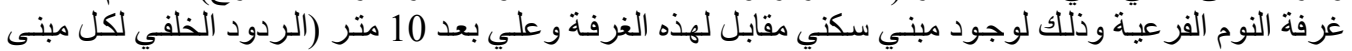


جدول (2): يوضح التوجيهات المختلفة للنموذج السكني المختار.

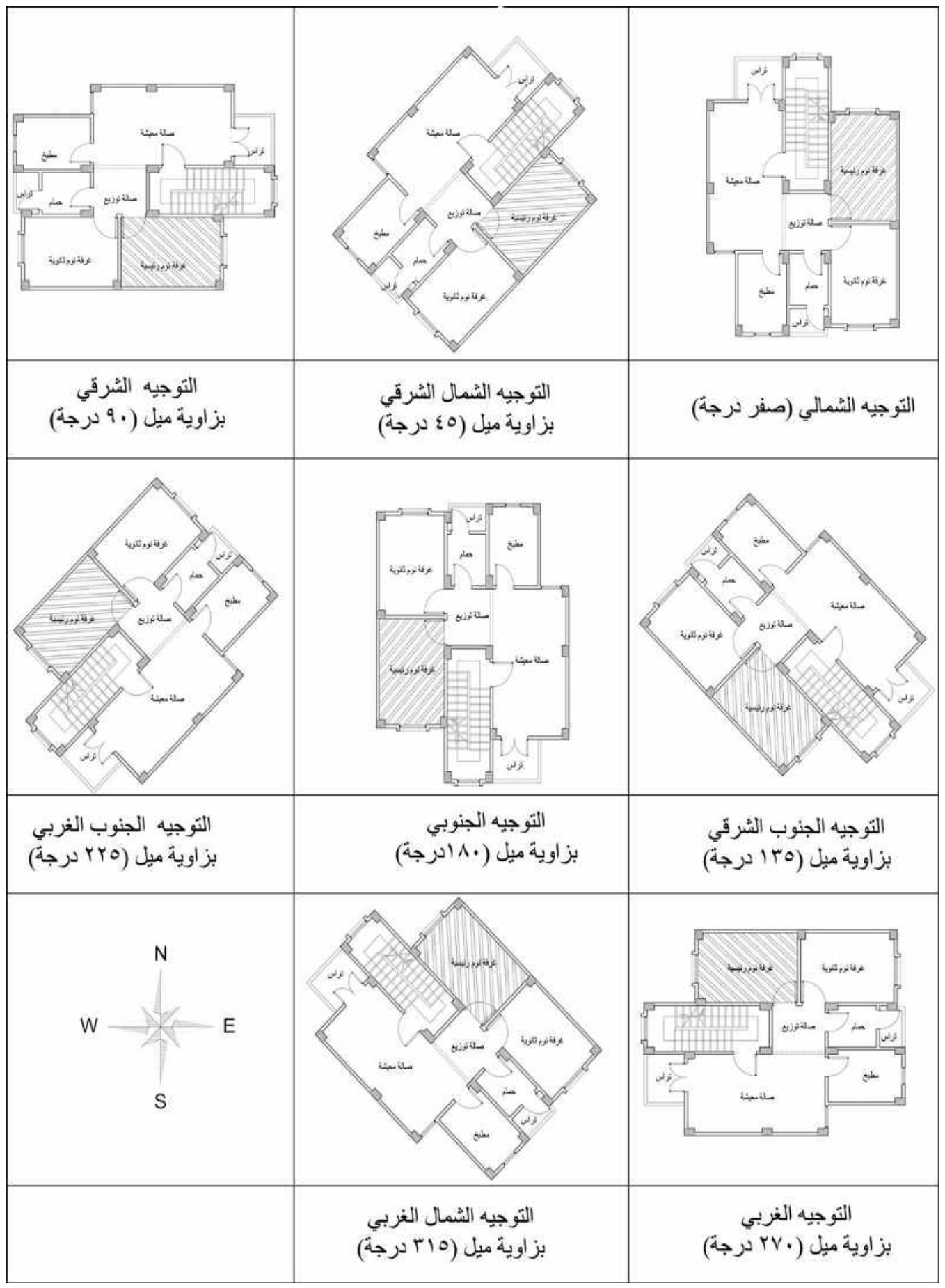




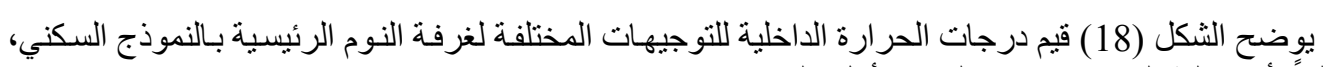
علماً بأن عملية المحاكاة تتم في الدور الأول درات بالمبني.
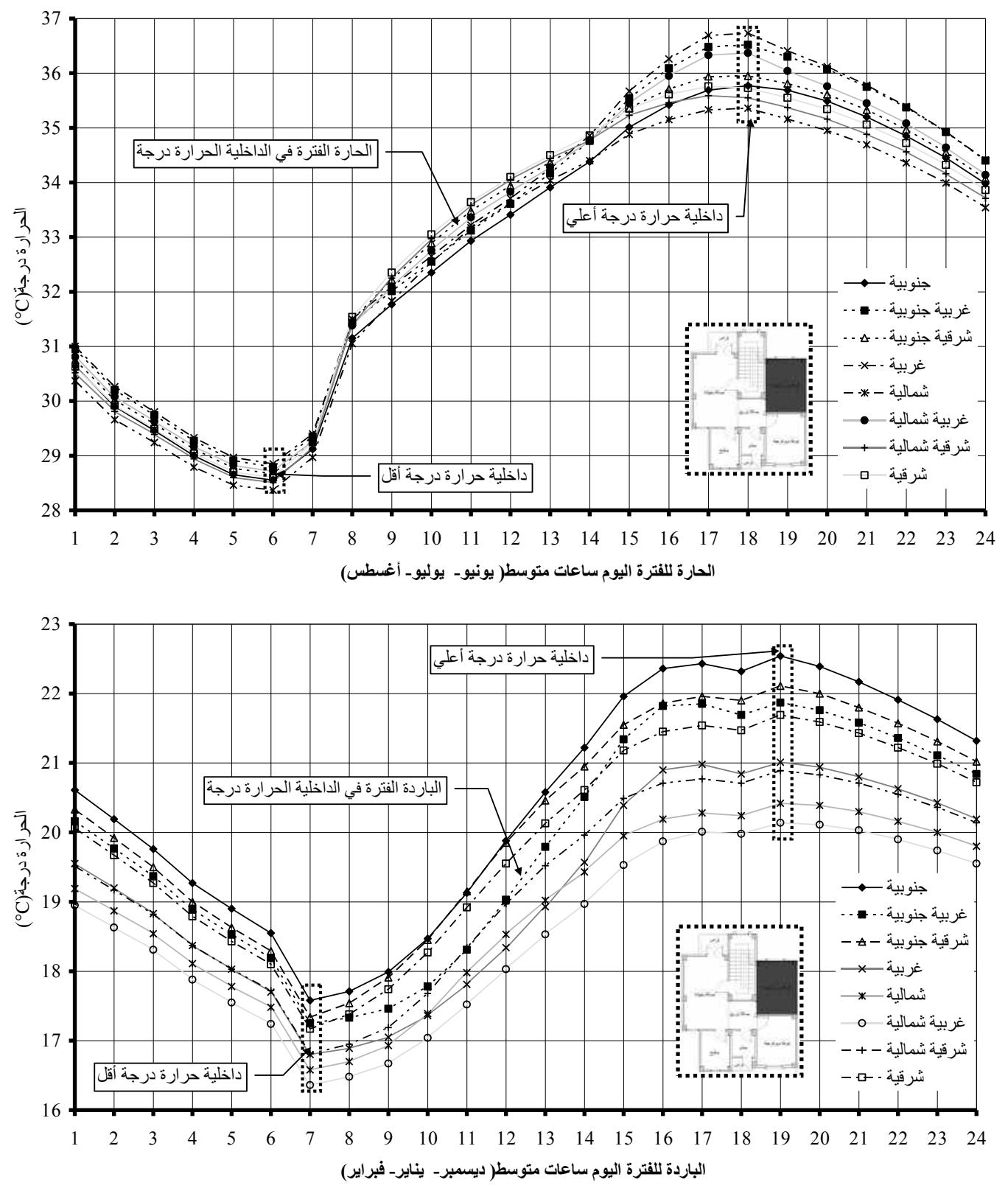

شكل (18): يوضح درجات الحر ارة الداخلية للتوجيهات المختلفة لغرفة النوم الرئيسية بالنموذج السكني للفترة الحارة و الباردة. 


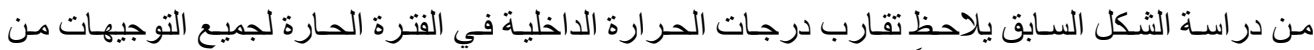

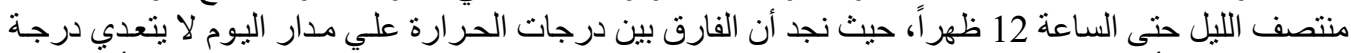

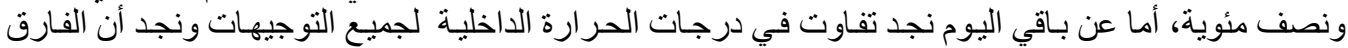

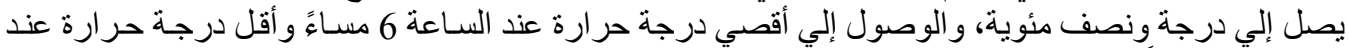
الساعة 6 صباحاً.

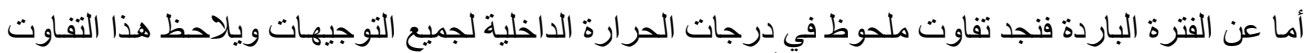

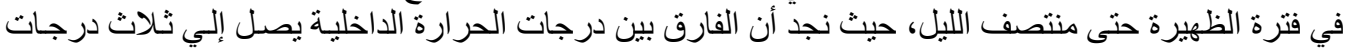

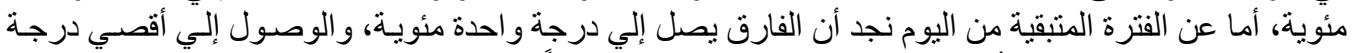

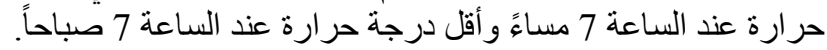

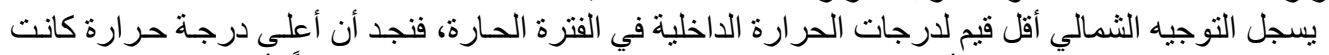

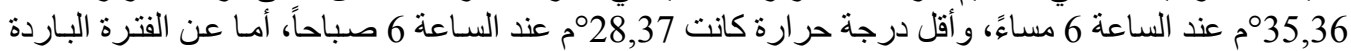

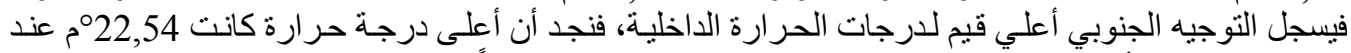

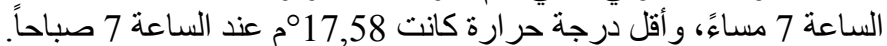

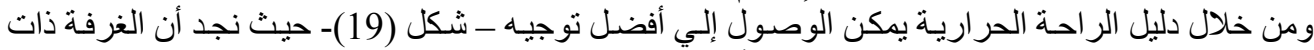

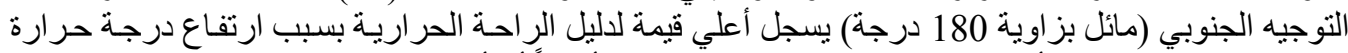

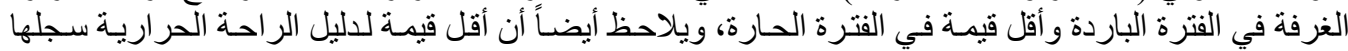
التوجيه الثمال الغربي (مائل بزاوية فئل فئسة 315 درجة).

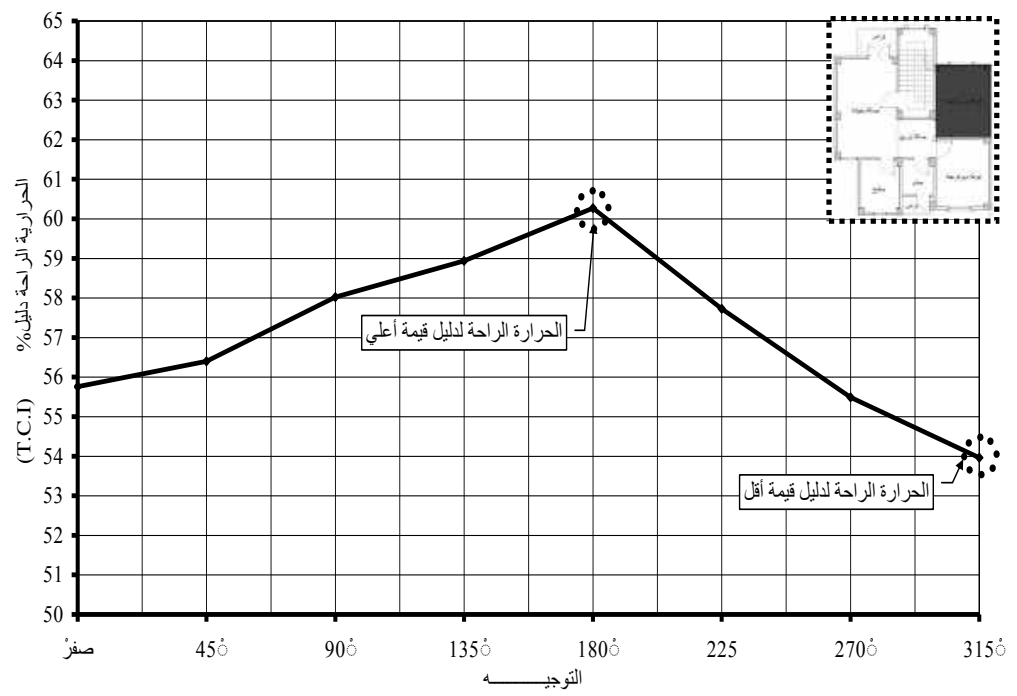

شكل (19): يوضح دليل الراحة الحرارية وتوجيه غرفة النوم الرئيسية. 
6- التتائج والتوصيات:

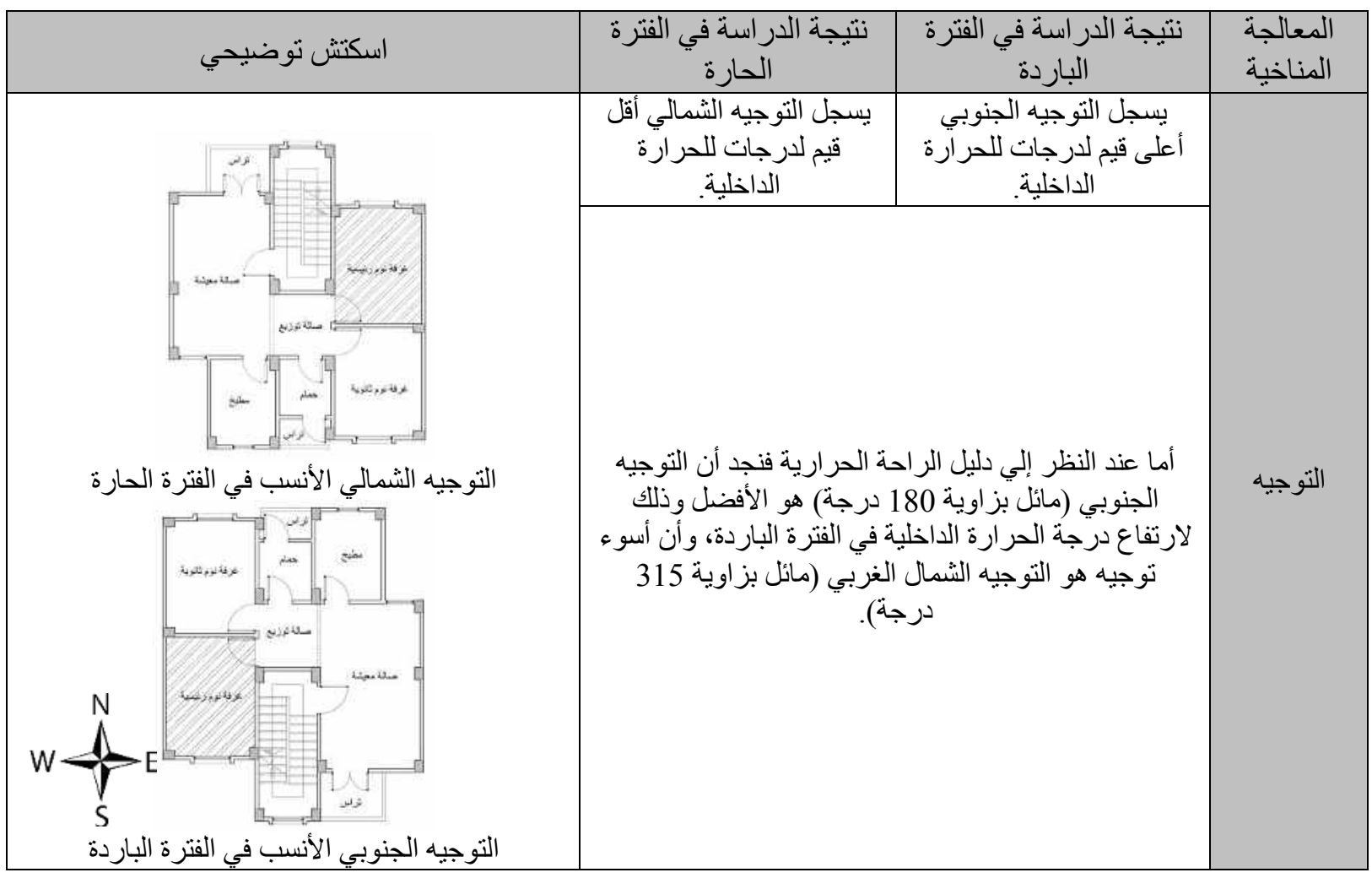

مما سبق توصي الدر اسة بالتالي:

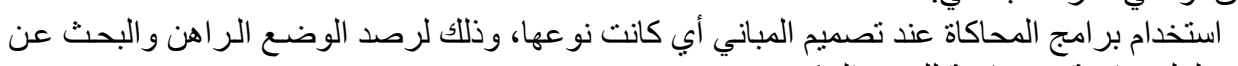
حلول مناخية ومعمارية للمبنى السكني.

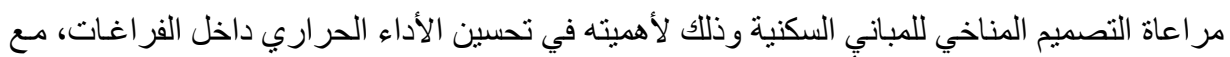

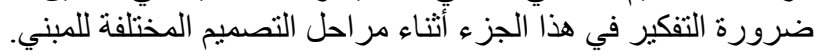

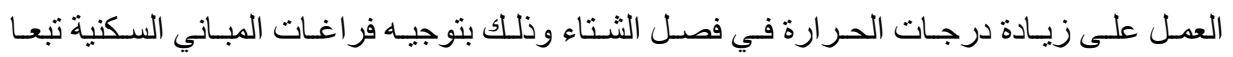

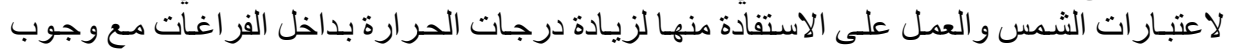

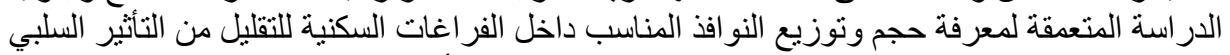

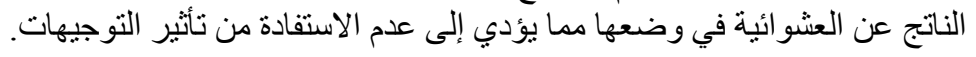

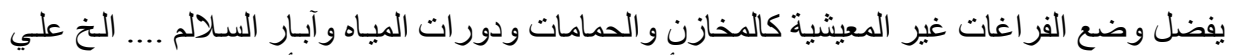

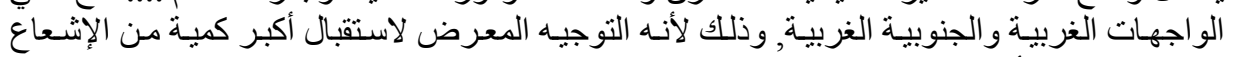

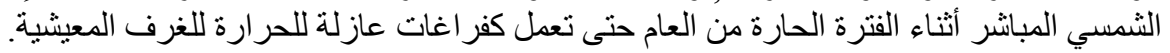

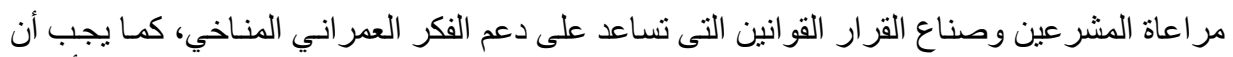

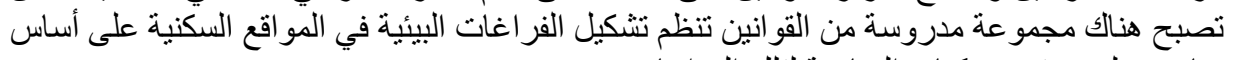

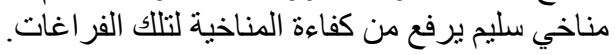

\section{7- المراجع العربية والأجنبية:}

1- أحمد هلال محمد: دراسـة تحليلية عن تأثير العوامل البيئيسة على تصميم المسكن في المدينة المصرية

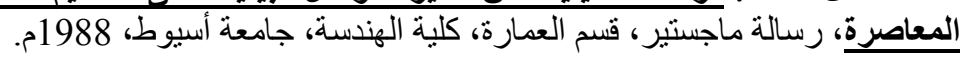


2- الهيئة العامة للأرصاد الجوية، الأطلس المناخى لمصر، وزارة النقل و المواصلات، جمهورية مصر العربية، 1996م

3- الهيئة العامة للتخطيط العمر اني، التخطيط الهيكلى للتجمع العمرانى الجديد بالوادي الأسيوطي، 1996م.

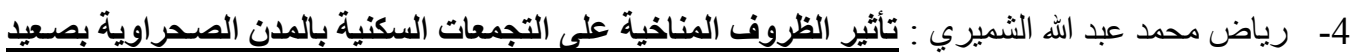

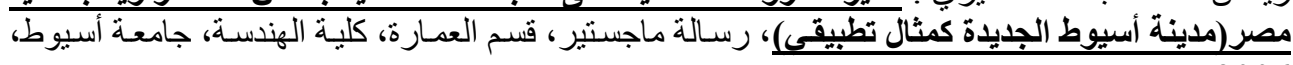
2006

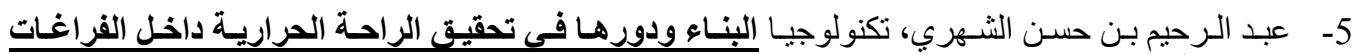

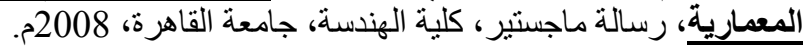

6- عبد المسيح يوسف عشي، المعايير التصميمية للأفنية الداخلية في العمارة العربية، رسالة دكتور اهاه، كلية الهندسة، جامعة القاهرة، فبر اير المعبر 1999.

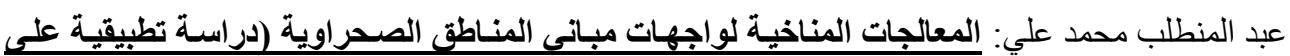

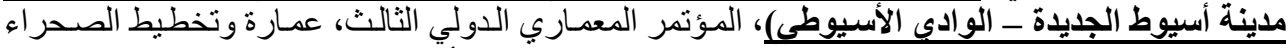

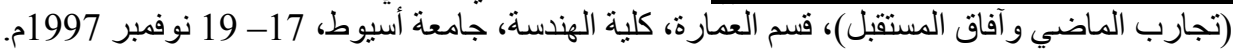

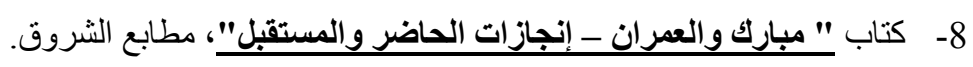

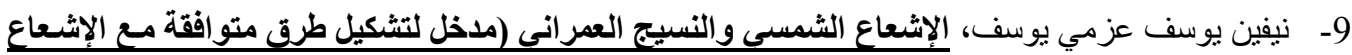

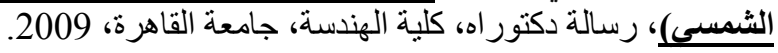

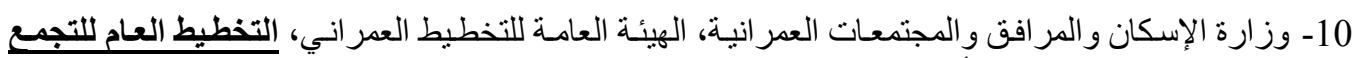

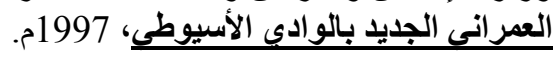

11- Flamant G., Heijmans N., Guiot E, Determination of the energy performances of ventilated double facades by the use of simulation integrating the control aspects Modelling aspects and assessment of the applicability of several simulation, Belgian Building Research Institute, Ministry of Economic Affairs Project, December 2004.

12- James Y. P. Lee, BASc, LEED AP, Sustainable Solution To Building Mechanical System - Simulation Of Thermo-Active Slab With Thermal Mass Using TAS, Earth Tech Canada Inc., Global Facility and Infrastructure, Mechanical Engineering Vancouver, British Columbia, Germany, 2010.

13- Konya,A.: Design Primer for Hot Climates, The Architectural Press Ltd, London, 1980.

14- Salwa Abdel Moneim El-Ginidy, The Effect of Building Envelope Design On Energy Conservation, Mc. S. thesis, Cairo University ,Egypt, February 2010.

15- Shick, W.L., Effects of building orientation on energy savings, Small homes council- Building Research Council, University of Illinois, Champaign, IL 61820.

16- Tarek Galal Habib, Trains of Urban Development in Egypt, Update Evaluation for the Experience of New Urban Communities, Ph. D., Faculty of engineering, University of Assiut, 2000.

17- http://www.edsl.net/main/

18- http://www.edsl.net/main/Support/Documentation.aspx

19- TAS Building Designer Software (EDSL Tas Version 9.2.0) 